This item was submitted to Loughborough's Institutional Repository (https://dspace.lboro.ac.uk/) by the author and is made available under the following Creative Commons Licence conditions.

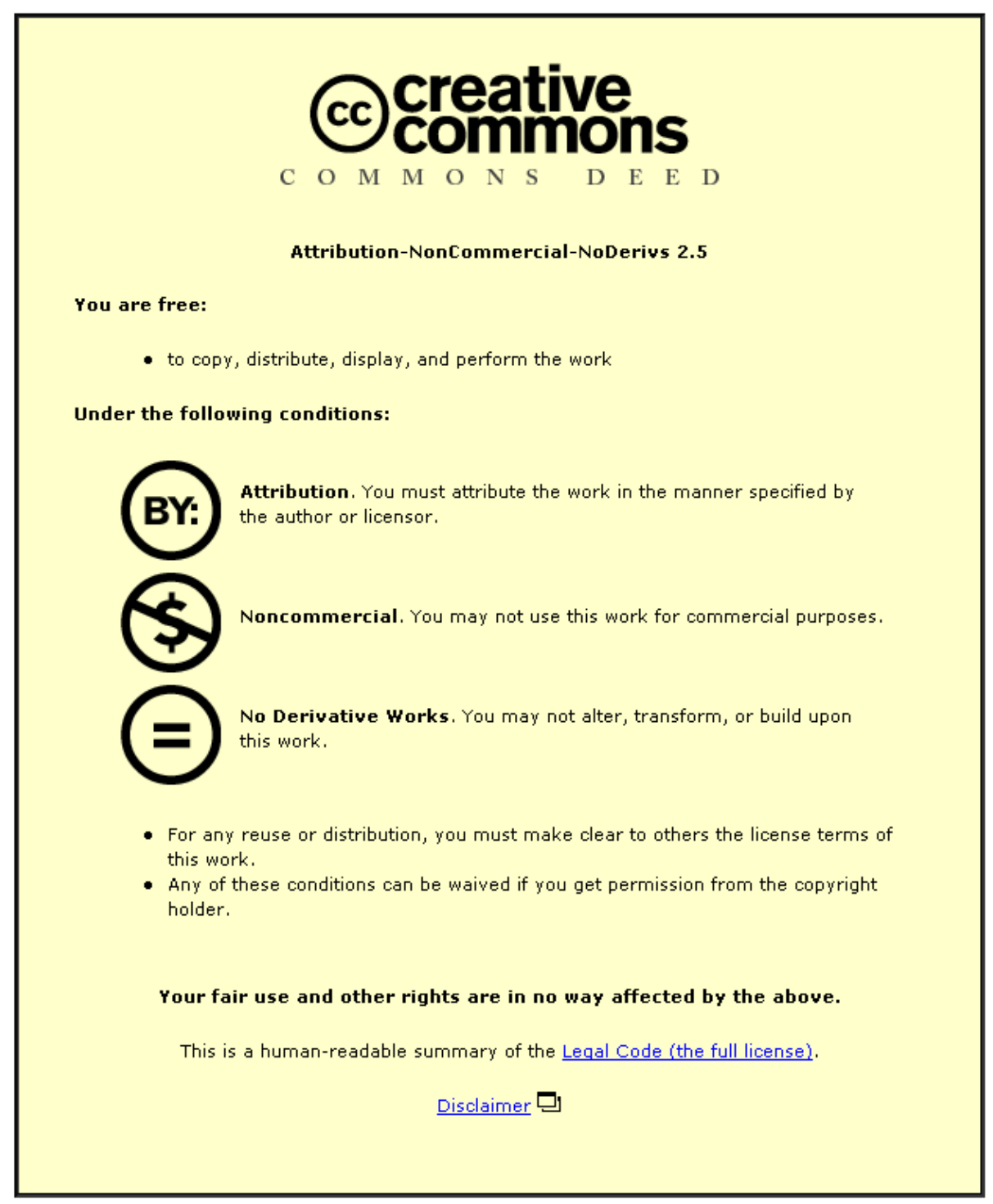

For the full text of this licence, please go to: http://creativecommons.org/licenses/by-nc-nd/2.5/ 


\title{
Resource Allocation And Block Coding Within A Three-Stage Collaborative Broadband Relay Network
}

\author{
S.K. Kassim, N.M. Eltayeb and J.A. Chambers \\ Advanced Signal Processing Group, Dept of Electronic and Electrical Engineering, Loughborough University, LE11 3TU,UK \\ Email: (s.k.kassim,n.e.eltayeb,j.a.chambers)@lboro.ac.uk
}

\begin{abstract}
In this work we propose a power control algorithm for a multi-input multi-output orthogonal frequency division multiplexing (MIMO-OFDM) multi-hop collaborative relaying network. Using orthogonal and quasi-orthogonal block codes with three stage processing our algorithm optimally distributes available transmission power based on the architecture and the channel condition at each stage so as to minimize the end-to-end bit error rate (BER) of the entire relay network. For high data rate applications with maximum throughput (as expected for future OFDM systems), we employ a regenerative relaying process, where the relays at each stage decode and through collaborative processing, re-encode the received data before onward transmission to the next stage and then to the destination.

We provide simulation results ${ }^{1}$ that confirm the performance improvement in the end-to-end bit error rate (BER) of the network using our explicit power allocation algorithm as compared with equal power distribution technique.
\end{abstract}

\section{INTRODUCTION}

In next generation wireless communication systems there will be an increasing demand for wireless systems with high quality of service $(\mathrm{QoS})$ and data capacity specification. Orthogonal frequency division multiplexing (OFDM) modulation and multi-stage transmission have been identified as two important techniques for these networks [1]-[2].

In a multi-stage communications, source terminals are allowed to communicate with the destination via relay terminals, this provides a flexible extension of the point-to-point system and a logarithmic increase in capacity as a function of numbers of relay stages [3]. This advantage could be further enhanced if closely spaced "idle" single antenna relay terminals cooperate together to form a relaying stage thereby creating a virtual multi-input multi-output (MIMO) channel [4] and mimics the performance advantage of MIMO, this concept is termed virtual antenna arrays (VAAs). It produces relaying links which suffer uncorrelated fading paths from the source to the destination, a condition required to exploit fully the advantage of a multiple antenna system. VAAs provide a better framework both for deployment of appropriate coding techniques over temporal, spatial and frequency dimensions with optimal power distribution to minimize the effect of

\footnotetext{
${ }^{1}$ Note that the results presented here form part of the result presented in [16]
}

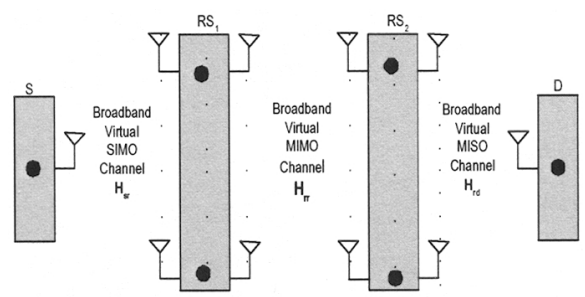

Fig. 1. Simple three-stage collaborative broadband relay architecture

fading in the network, due to the local communication between the relay nodes [5].

These earlier works have considered relaying networks formed over narrowband channels (frequency flat) and hence are only generally appropriate for low data rate transmission.

However, for higher data rate application over broadband frequency selective fading channels, there becomes a clear need for researchers to focus attention on extending the advantages offered by multi-stage networks to future broadband systems.

Although OFDM has become the de-facto approach to broadband transmission over frequency selective fading channels, largely because of its higher spectral efficiency in wireless broadband systems, there are still problems of power saving for extra-high data rate transmission with QoS constraints and its vulnerability to radio propagation and synchronization errors [6]. One way to overcome these problems is by employing a multi-stage communication technique, which takes advantage of relay nodes to obtain additional diversity gain [6].

To exploit fully the benefit offered by multi-stage communication over broadband channels, effective use of both radio and power resources (especially in a constrained situation) has been identified as one major issue to be addressed. This will further minimize the end-to-end bit error rate (BER), and as such has been receiving considerable research attention lately such as the work presented in [7]. As with the narrowband application, they have limited their analysis to a two stage network, which offers limited capacity [3] and coverage area as compared to networks with higher number of stages.

The work presented in this paper is based on the VAA concept as its provides an excellent framework for optimal 
power resource allocation and provides a further attempt to effectively use these "scarce" network resources, for an increased QoS performance and thereby further enhance the coverage area of the previously proposed network (without jeopardizing performance). We demonstrate the advantages of different orthogonal and quasi-orthogonal coding schemes at the relaying stages on the performance of the network and show that with effective explicit power allocation over stages and frequency a significant improvement in the end-to-end BER performance can be achieved. We use the time division multiple access (TDMA) technique, where the total frame duration is divided optimally among each relaying stage, we also assume that for a high data rate application and better throughput the relays at each stage decode the received data to remove the effect of noise before forwarding to the next stage. Finally, we assume a fixed power budget for the entire network and that the channel fading characteristic is quasistatic, hence we consider a frequency selective slow fading channel.

The organization of this paper is as follows. Section II describes our MIMO-OFDM multi-stage system model and the general structure for rate one full diversity space frequency orthogonal, quasi-orthogonal and extended orthogonal codes. In section III we discuss the power allocation strategies while our simulation results are discussed in section IV and finally some closing remarks are presented in section $\mathrm{V}$.

\section{SYSTEM MODEL}

As shown in Figure 1, the communication between the source $(S)$ and the destination $(D)$ is achieved through two sets of relaying stages $R S_{1}$ and $R S_{2}$, each having $k$ number single antenna relaying elements The source and the destination are each made up of a single antenna. We assume a quasistatic frequency selective Rayleigh fading channel between each stage, and direct communication between the source and destination is not possible. Denoting the channel from the source to the first relaying stage as $H_{s r}$, that from the first stage to the second stage as $H_{r r}$ and from the second stage to sink as $H_{r d}$. These channels are exactly known by the receivers of each stage but the transmitter may or may not have knowledge of the full channel state information (CSI).

Let $\mathbf{x}_{i}^{j}$ denote the data stream transmitted within the $i^{\text {th }}$ stage from the $j^{\text {th }}$ relay (which would be 1 in the first stage and $1, \ldots \ldots, k$ in the second and third stages), transmitted over a frequency selective fading channel having $L$ independent channel taps, where $\mathbf{x}_{i}^{j}=\left[x_{i}^{j}(0) x_{i}^{j}(1) \ldots x_{i}^{j}(N-1)\right]^{T}$, where the $n^{\text {th }}$ element of the vector is the $n^{\text {th }}$ data symbol and indeed the sub-carrier index, $(.)^{T}$ is a transpose operator and $N$ is the frame length.

Let us denote the complex channel transfer functions of the subcarrier $n$ from the source $S$ to the $j^{\text {th }}$ relay of $R S_{1}$ as $H_{j}^{1}(n)$, from the $m^{\text {th }}$ relay within $R S_{1}$ to the $j^{\text {th }}$ relay within $R S_{2}$ as $H_{m j}^{2}(n)$ and that of the $m^{\text {th }}$ relay within $R S_{2}$ to the destination as $H_{j}^{3}(n)$, note that with each stage, $m$ is used to index the transmitting antennas whilst $j$ indexes the receiving antennas. With these notations, $H_{j}^{1}(n)=$
$\sum_{l=0}^{L-1} h_{j}^{1}(l) e^{-j 2 \pi l n / N}, H_{m j}^{2}(n)=\sum_{l=0}^{L-1} h_{m j}^{2}(l) e^{-j 2 \pi l n / N}$ and $H_{j}^{3}(n)=\sum_{l=0}^{L-1} h_{j}^{3}(l) e^{-j 2 \pi l n / N}$, where $h_{j}^{1}, h_{m j}^{2}$ and $h_{j}^{3}$ are their corresponding channel impulse response coefficients. The receive signal on each sub-carrier by the $j^{\text {th }}$ receiving antennas of the $i^{\text {th }}$ stage can be expressed as $y_{i}{ }^{j}(n)=\mathbf{x}_{i}{ }^{m}(n) \mathbf{h}_{i}{ }^{m}(n)+z_{i}{ }^{j}(n)$. where $\mathbf{h}_{i}{ }^{m}(n)=$ $\left[H_{i}^{1}(n) H_{i}^{2}(n) \ldots . . H_{i}^{T_{i}}(n)\right]^{T}$ with $T_{i}$ representing the total number of transmitting relays of the $i^{\text {th }}$ stage and $z_{i}{ }^{j}(n)$ is the received noise of the $j^{\text {th }}$ receiver, which is assumed to be an independent and identically distributed (i.i.d.), zero mean and unit variance complex Gaussian noise variable at the receive antenna, and $\mathbf{x}_{i}{ }^{m}(n)=\left[x_{i}^{1}(n) x_{i}^{2}(n) \ldots . . x_{i}^{T_{i}}(n)\right]$.

The received signals $y_{i}{ }^{j}(n)$ at the first stage (when $i=1$ ) are combined using a maximum ratio combiner (MRC) and distributively re-encoded (assuming full cooperation among the relays) at $R S_{1}$ and $R S_{2}$, depending on the cooperating relays and the preferred coding methods, using either Alamouti space frequency block codes (A-SFBC), quasi-orthogonal space frequency block codes (QO-SFBC) and extended orthogonal space frequency block codes (EO-SFBC). The same encoding is also performed at these stages and their inputoutput relationship is presented in the next section.

\section{A. Space Frequency Orthogonal, Quasi-Orthogonal and Extended-Orthogonal Block Codes}

In [8] an example of coding over a frequency selective channel is presented based on the Alamouti space time block code (A-STBC), which have the coding matrix

$$
\mathbf{X}_{2 X 2}=\left[\begin{array}{cc}
x_{1}\left(n_{0}\right) & x_{2}\left(n_{0}\right) \\
-x_{2}^{*}\left(n_{1}\right) & x_{1}^{*}\left(n_{1}\right)
\end{array}\right]
$$

where $x_{i}(n), i=1,2$ represent the space frequency symbols and $(.)^{*}$ denotes complex conjugate.

This codeword is proportional to a unitary matrix and provides maximum possible diversity with full rate transmission over two transmit and $N_{r}$ received antennas. If we assume that the channel remains constant over two consecutive subcarriers i.e. $H\left(n_{0}\right)=H\left(n_{1}\right)$ then the receiver can detect the received symbols over two sub-carriers without intersymbol interference with a linear symbol-wise operation.

Replacing the time index with OFDM sub-carrier index in the codeword presented in [9] and [10], we can express a new class of codes in terms of spatial and frequency dimensions and call them quasi-orthogonal space frequency block code (QO-SFBC) and extended orthogonal space frequency block code (EO-SFBC) respectively. We represent the codeword matrix for QO-SFBC as

$$
\mathbf{X}_{4 X 4}=\left[\begin{array}{cccc}
x_{1}\left(n_{0}\right) & x_{2}\left(n_{0}\right) & x_{3}\left(n_{0}\right) & x_{4}\left(n_{0}\right) \\
-x_{2}^{*}\left(n_{1}\right) & x_{1}^{*}\left(n_{1}\right) & -x_{4}^{*}\left(n_{1}\right) & x_{3}^{*}\left(n_{1}\right) \\
-x_{3}^{*}\left(n_{2}\right) & -x_{4}^{*}\left(n_{2}\right) & x_{1}^{*}\left(n_{2}\right) & x_{2}^{*}\left(n_{2}\right) \\
x_{4}\left(n_{3}\right) & -x_{3}\left(n_{3}\right) & -x_{2}\left(n_{3}\right) & x_{1}\left(n_{3}\right)
\end{array}\right]
$$

and for EO-SFBC as

$$
\mathbf{X}_{2 X 4}=\left[\begin{array}{cccc}
x_{1}\left(n_{0}\right) & x_{1}\left(n_{0}\right) & x_{2}\left(n_{0}\right) & x_{2}\left(n_{0}\right) \\
-x_{2}^{*}\left(n_{1}\right) & -x_{2}^{*}\left(n_{1}\right) & x_{1}^{*}\left(n_{1}\right) & x_{1}^{*}\left(n_{1}\right)
\end{array}\right]
$$


where $n_{0}, n_{1}, n_{2}$ and $n_{3}$ denote four adjacent channel frequency indices.

These codeword matrices does not have the same property as equation (1), hence yielding coupling between the estimated symbols at the receiver and making decoding more complex, i.e. maximum likelihood decoding is pair-wise, as a result, there is a loss in diversity order offered by these coding schemes with full rate transmission over four transmit and $N_{r}$ received antennas.

An attempt to eliminate or reduce these coupling factors was presented in [11] and [12], using a feedback approach, wherein they pre-multiply the transmitted symbols from two antennas with a phase angle feedback from the receiver.

If we assume that the channel remains constant over two and four consecutive frequencies for EO-SFBC i.e. $\left[H\left(n_{0}\right)=\right.$ $\left.H\left(n_{1}\right)\right]$ and QO-SFBC i.e. $\left[H\left(n_{0}\right)=H\left(n_{1}\right)=H\left(n_{2}\right)=\right.$ $H\left(n_{3}\right)$ ], then the receiver can detect the received symbols over two/four sub-carriers and we can exploit the feedback approaches developed in [11] and [12] to orthogonalize the resulting channel matrix. This collapses the resulting vector detection problem into scalar detection problem and we employ the optimum maximum likelihood detection technique to extract the transmitted signal.

Using the above coding schemes the received signal at the second and the third stage on the two/four adjacent subcarriers, at the $j^{\text {th }}$ received antenna can be expressed as

$$
\mathbf{y}_{i}^{j}=\mathbf{X}_{i}{ }^{m} \mathbf{h}_{i}{ }^{m}+\mathbf{z}_{i}^{j}, i \in(2,3)
$$

dropping the stage and receiver indices in the vector elements, we then express $\mathbf{y}_{i}{ }^{j}=\left[y\left(n_{0}\right) y\left(n_{1}\right) \ldots \ldots y\left(N_{f-1}\right)\right]^{T}$, and $\mathbf{z}_{i}{ }^{j}(n)=\left[z\left(n_{0}\right) z\left(n_{1}\right) \ldots \ldots z\left(N_{f-1}\right)\right]^{T}$, where $N_{f}$ is the number of sub-carriers in one block.

The vector $\mathbf{h}_{i}{ }^{m}=\left[H^{1}(n) H^{2}(n) \ldots . . H^{T_{i}}(n)\right]^{T}$ since $H(n)$ is quasi static over $N_{f}$ sub-carriers and $\mathbf{X}_{i}{ }^{m}$ is the corresponding codeword matrix in equations (1), (2) or (3) for (A-SFBC), QO-SFBC and EO-SFBC.

Note in this work we do not include the additional complexity of the grouping scheme proposed in [13] to maximally exploit both the spatial and temporal diversity of the MIMOfrequency selective channels. Instead we assume that in a practical realization of our scheme, convolution encoding and interleaving could be added, therefore all our simulations will be presented in the uncoded sense.

\section{OPtimum POWER ALlOCATION STRATEGY}

In this section, optimum power allocation within the entire network is investigated. If the total power available to transmit information from the source to destination is $P$, an optimal inter-stage power allocation scheme presented in [4], is extended to frequency selective Rayleigh fading channels. Since OFDM is a multi-carrier transmission technique, to further improve the performance of the system, we present an optimal intra-stage power allocation strategy over each sub-carrier in addition inter-stage allocation. It is worth noting that at this stage we assume that the channel at each stage is known to the transmitter at that stage, which is feasible in a time division duplex (TDD) system where the up and down-link channel can be assumed identical [14].

\section{A. Equal Power Equal Sub-carrier Gains}

Here $P$ is first allocated equally to each stage such that the power allocated to the $i^{\text {th }}$ stage can be expressed as

$$
P_{i}=\frac{P}{K}
$$

where $K$ is the number of relaying stages.

With $N_{T}$ transmit antennas, the power allocated to each transmitting antennas of the $i^{\text {th }}$ stage is expressed as

$$
P_{i}[t]=\frac{P_{i}}{N_{T}}, t \in\left(1, \ldots, T_{i}\right)
$$

where $T_{i}$ depends on the number of cooperating transmit relay elements (in our case 2 or 4 )

For OFDM transmission, let $\left\{P_{t, j}(n)\right\}_{n=0}^{N-1}$ and $\left\{\lambda_{t, j}(n)\right\}_{n=0}^{N-1}$ denote the transmitted power in each subcarrier and their corresponding channel gain squared from transmitting antenna $t$ to the receiver $j$ of the $i^{t h}$ stage respectively. The transmit power allocated to the $n^{\text {th }}$ subcarrier between the transmit antenna $t$ and any receive antenna $j$ can be expressed as (dropping subscript $j$ for easy of representation)

$$
P_{t}(n)=\frac{P_{i}[t]}{N}
$$

where $N$ is the total number of sub-carriers.

\section{B. Un-equal Power Equal Sub-carrier Gains}

Here $P$ is distributed among the relaying stages such that

$$
P=\sum_{i=1}^{K} P_{i}
$$

An optimum $P_{i}$ that minimizes the end-to-end bit error rate (BER) can be expressed from [4] as

$$
P_{i}=\left[\sum_{j=1}^{K} \alpha_{j}\left(\frac{q_{i}^{-1} A_{i}^{-1} B_{i}^{q_{i}}}{q_{j}^{-1} A_{j}^{-1} B_{j}^{q_{j}}}\right)^{\frac{1}{q_{\max +1}}}\right]^{-1}
$$

where $q_{i}=t_{i} . r_{i}$ which is the product of the number of transmit and receive antennas of the $i^{\text {th }}$ stage, $q_{\max }=$ $\arg \max \left(q_{1}, \cdots, q_{k}\right)$. The parameter $\alpha_{j}$ is the fractional frame duration which for our TDMA system satisfies $\sum_{i=1}^{K} \alpha_{i}=1$. The $i^{\text {th }}$ stage fractional frame duration can be expressed as

$$
\alpha_{i}=\frac{\prod_{j=1, j \neq i}^{K} C_{j} \cdot \log _{2}\left(M_{j}\right)}{\sum_{k=1}^{K} \prod_{j=1, j \neq k}^{K} C_{j} \cdot \log _{2}\left(M_{j}\right)}
$$

with $A_{i}$ and $B_{i}$ expressed as

$$
\begin{gathered}
A_{i}=\frac{2 q_{i}}{\log _{2}\left(M_{i}\right)} \\
B_{i}=\frac{G Q A M_{i}}{R_{i}} \frac{\gamma_{i}}{t_{i}} \frac{S}{N}
\end{gathered}
$$


where $G Q A M=3 / 2 /\left(M_{i}-1\right), \gamma_{i}$ is the total channel gains of the $i^{\text {th }}$ stage, $q_{i}=1-1 / \sqrt{M_{i}}, C_{i}$ is the code rate of the $i^{t h}$ stage while $M_{i}$ is the modulating index and $\frac{S}{N}$ is the $i^{t h}$ stage receiver signal to noise ratio. This power is allocated equally among each transmitting antenna of each stage which is further distributed equally among each sub-carrier from the $t^{t h}$ transmitting antenna, in accordance with equation (7).

\section{Un-equal Power Un-equal Sub-carrier Gains}

As shown in [4], the optimum power allocation strategy that minimises the end-to-end bit error rate (BER) is one that distributes the power based on the architecture of each stage such that the worst stage (which determines the performance of the entire network) obtains the greatest percentage of the total power to improve its performance and hence the entire network, Therefore, $P_{i}$ obeys equation (9).

As noted in [15], the well known Shannon's water filling algorithm is the optimal power allocation method for an orthogonal carrier system, but its capacity advantage disappears at larger signal-to-noise ratios (SNRs) [16]. Here we propose an explicit approximation of this algorithm over each subcarriers, in order to improve the average BER performance of the network over a larger range of SNR.

If $P_{i}[t]$ is the total transmitted power from the $t^{\text {th }}$ transmitter of the $i^{\text {th }}$ stage, then the power allocated to the $n^{\text {th }}$ sub-carrier between the transmit antenna $t$ and any receiver antenna $j$ can be expressed as,

$P_{t}(n)=\left(\frac{\prod_{g=1, g \neq n}^{N} \lambda_{t}[g]^{-\frac{1}{2}}}{\sum_{w=1}^{N} \prod_{g=1, g \neq w}^{N} \lambda_{t}[g]^{-\frac{1}{2}}}\right) P_{i}[t], n=(0,1, \ldots, N-1)$

Note if $P_{i}$ is distributed in accordance with equation (6), therefore $P_{i}$ can be expressed as

$$
P_{i}=\sum_{t=1}^{T_{i}} \sum_{n=0}^{N-1} P_{t}(n), T_{i} \in Z^{+}
$$

This power allocation scheme is designed for quasi-static channels.

\section{SIMULATION RESULTS}

In Figures 2, 3 and 4, we show the end-to-end (average) BER performance of the proposed three stage relaying system with two and four transmit/receive antennas at the second stage when the proposed resource allocation schemes are applied. We evaluate the end-to-end bit error rate (BER) as a joint probability of the BER of each stage. For each stage we assume the channel between the transmitter $t$ and the receiver $j$ is length three with each coefficient a zero mean circular complex Gaussian random variable with equal variance, normalised so that $\sum_{l=0}^{2} \sigma_{l}^{2}=1$. Also we assume 64 length OFDM symbols and a cyclic prefix of length 8 . The received symbols at the first stage are always combined using a maximum ratio combiner (MRC).

Figure 2 presents results for open-loop space frequency Alamouti encoding over the second and third stages. Figure 3 and 4 assumes channel state information (CSI) is available

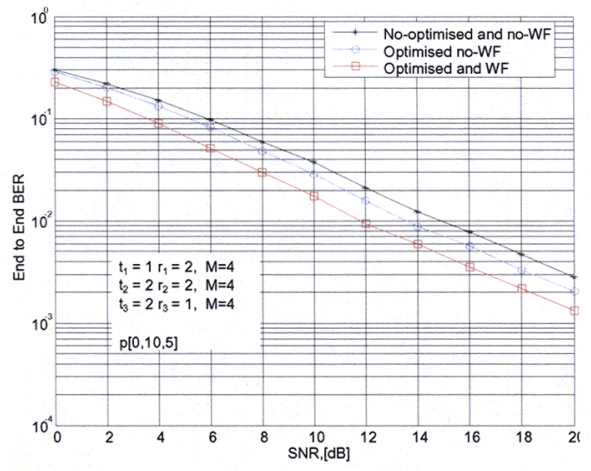

Fig. 2. End-to-end BER OSF-OFDM as a function of SNR.

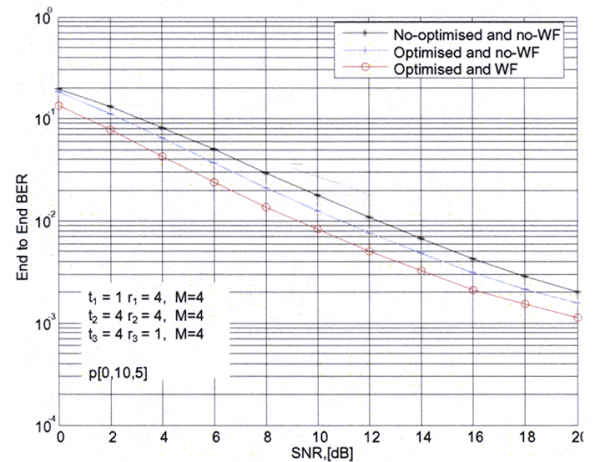

Fig. 3. End to End BER CLQO-SFBC-OFDM as a function of SNR.

in the transmitters of stages two and three and exploits a closed-loop quasi-orthogonal space frequency block coding (CLQO-SFBC) whilst Figure 4 exploits a closed-loop extended orthogonal space frequency block coding (CLEO$\mathrm{SFBC}$ ) in the second and third stages.

Note that in the figures, the "No-optimum and no-WF" represents equal power allocation across stages and subcarriers, "optimized no-WF" represents optimized interstage allocation with equal sub-carrier power and "optimized and WF" represents optimized interstage and sub-carrier power allocation using our strategy. Also our simulation result is parameterized by $p$ which defines each link gain with respect to the first stage, e.g $p(0,10,5)$ means that the second link is 10 times stronger than the first one and the last link is 5 times stronger than the first one. Although this last stage has a 5 fold increase relative to the first stage, the first stage still performs better than the last stage because of its inherent array gain advantage, and with equal power distribution across stages, the radiated power $P_{1}$ in the first stage is $T_{3}$ times higher than the power radiated from individual antennas of the $3^{\text {rd }}$ stage, where $T_{3}$ is the number of transmitting relays at the third stage.

As can be seen from the figures, our proposed resource allocation strategy out-performs equal power allocation strat- 


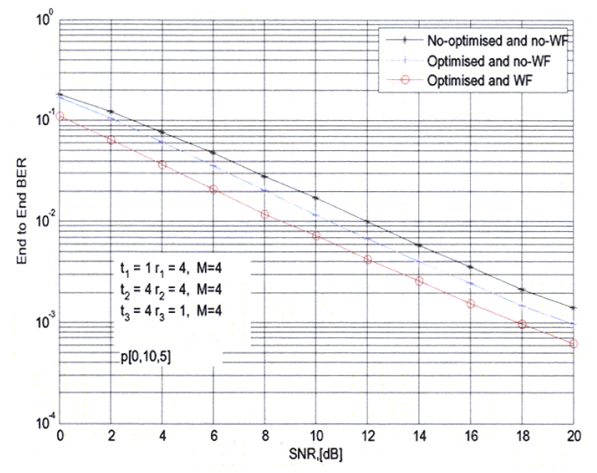

Fig. 4. End to End BER CLEO-SFBC-OFDM as a function of SNR.

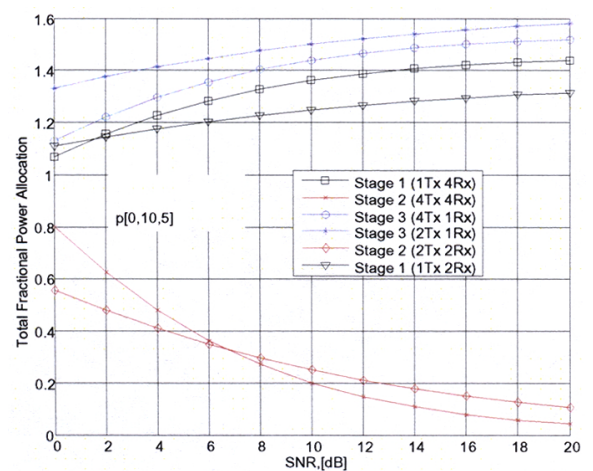

Fig. 5. Optimum Inter-stage Power Control as a function of SNR.

egy over each sub-carrier. It also shows that the performance of inter-stage power allocation over broadband multi-stage communication can further be enhanced by optimum subcarrier resource allocation. For instance in Figure 2, at end-toend BER of $10^{-2}$ our proposed optimum intra-stage allocation combined with optimal interstage allocation out-performs equal power allocation by approximately $3 d B$ and there is a loss of approximately $1.5 \mathrm{~dB}$ if not employed with optimal interstage allocation. However, in a system using CLQOSFBC, a $3.5 d B$ gain is achieved at the same BER while for a system with CLEO-SFBC a $3.5 d B$ gain is achieved. However, looking at Figures 3 and 4, at $20 d B$ the CLEO-SFBC enjoys additional benefit of a maximum coding gain -diversity and transmit array gain of $0.5 \times 10^{-3}$ over CLQO-SFBC, these benefits are also evidenced with both our inter and intra stage power allocation. Figure 5 shows the variation of interstage power allocation over the SNR range considered. It can be seen that on the average, the third stage which is the weakest link is allocated more power in order to boost its performance, for instance with a $1 \times 4,4 \times 4$ and $4 \times 1$ configuration, the power allocated to each stage at $10 d B$ is $1.3612,0.2002$ and 1.4386 respectively and likewise for $1 \times 2,2 \times 2$ and $2 \times 1$ is $1.5007,0.2499$ and 1.2494 respectively. In both cases the total power available to the network is constrained to $K=3$ and the interstage allocation obeys equation (8).

\section{CONCLUSION}

In this work we have presented a three-stage relaying structure which has the aim of increasing the coverage area of a broadband communication system (without jeopardizing its performance).

We have confirmed the advantages of using different space frequency coding schemes over the network in order to combat the effect of multi-path propagations between the source and through various relaying stages to the destination. We then extended the interstage power allocation for a twostage communication over a frequency non-selective channel presented in [4] to a three-stage communications over a frequency selective channel and proposed a further optimal power allocation algorithm across each OFDM sub-carrier at each relaying stage. Our results showed a considerable end-toend BER advantage when compared to equal power allocation on each stage.

\section{REFERENCES}

[1] R.Van Nee and R. Prasad, "OFDM for wireless multimedia communications," Artech House: 2000

[2] L. Le and E. Hossain, "Multihop cellular networks: potential gains, research challenges and a resource allocation framework," IEEE Communication Magazine, Vol. 45, pp.66 - 73, Sept., 2007.

[3] M. Gastpa and M. Vetterli, "On the capacity of wireless network: The relay case," in Proc. IEEE Computer and Communication Society Conf. (INFOCOM), pp.1577 - 1855, 2002.

[4] M. Dohler, "Virtual antenna arrays" PhD thesis, University of London, Nov., 2003.

[5] S.K. Kassim, M. Hayes, N.M. Eltayeb and J.A. Chambers, "Exploitation of quasi-orthogonal space time block code in virtual antenna arrays: Part II monte carlo-based throughput evaluation ," in Proc. 67th IEEE Vehicular Technology Conf. (VTC), Vol. 5, pp.342 - 346, May, 2008.

[6] R. Pabst, B.H. Walke and D.C. Schultz, "Relay-based deployment concepts for wireless and mobile broadband radio," IEEE Communications Magazine, vol. 42, pp.80-88. Sep., 2004.

[7] J. Shi, G. Yu, Z. Zhang and P. Qiu, "Resources allocation in OFDM based multi-hop wireless networks ," in Proc. 63rd IEEE Vehicular Technology Conf. (VTC), Vol. 1, pp.319 - 323, May, 2006.

[8] A.A. Hutter, S. Mekraz, B.N. Getu and F. Platbrood, "Alamouti-based space-frequency coding for OFDM," J. Wireless Personal Communication, Vol. 35, pp.173-185, Oct., 2005.

[9] H. Jafarkhani, "A quasi-orthogonal space-time block code," IEEE Trans. Communications, Vol. 49, pp.1-4, Jan., 2001.

[10] Y. Yu, S. Kerouden and J. Yuan, "Closed-loop extended orthogonal space-time block codes for three and four transmit antennas," IEEE Signal Processing Letters, Vol. 13, pp.273-276, May, 2006.

[11] Toker C., Lambotharan S., and Chambers J.A., "Closed-loop quasiorthogonal STBCs and their performance in multipath fading Environments and When Combined With Turbo Codes," IEEE Trans. Wireless Communications, Vol. 3, pp.1890-1896, Nov., 2004.

[12] N.M. Eltayeb, S. Lambotharan, and J. Chambers, "A phase feedback based extended space-time block code for enhancement of diversity," in Proc. 65th IEEE Vehicular Technology Conf. (VTC), pp.2296-2299 April, 2007.

[13] G.B. Giannakis, Z Liu, X. Ma and S. Zhou, "Space time coding for broadband wireless communications," New Jersey: Wiley, 2007.

[14] M.Vu and A. Paulraj, "MIMO wireless linear precoding," IEEE Signal Processing Magazine, Vol., pp.86-105, Sept., 2007.

[15] T.M. Cover, and J.A. Thomas, "Element of information theory," New York: Wiley, 1991.

[16] S.K. Kassim, N.M. Eltayeb and J.A. Chambers, "Orthogonal and quasiOrthogonal block coding within three stage collaborative broadband relay networks," submitted to IET J. Communications, Dec., 2008. 